\title{
ON THE STABLE HOMOTOPY OF QUATERNIONIC AND COMPLEX PROJECTIVE SPACES
}

\author{
DAVID M. SEGAL ${ }^{1}$ \\ ABSTRACT. Let the image in $H_{l k}\left(\mathrm{QP}^{\times}: Z\right)=Z$ of stable homotopy \\ under the Hurewicz homomorphism be $h(k) \cdot Z$. Using the Adams \\ spectral sequence for the 2-primary stable homotopy of quater- \\ nionic and complex projective spaces it is shown that $h(k)$ is $(2 k)$ ! if \\ $k$ is even and is $(2 k) ! / 2$ if $k$ is odd.
}

1. Introduction. Let $Q P^{\infty}$ denote infinite quaternionic projective space. We prove

TheOREM 1.1. For $k>0$ the stable Hurewicz homomorphism

$$
\stackrel{\mathrm{S}}{\pi_{4 k}}\left(\mathrm{QP}^{\infty}\right) \rightarrow H_{4 k}\left(\mathrm{QP}^{\infty} ; Z\right) \cong Z
$$

maps onto $(2 k) ! \cdot Z$ for $k$ even, onto $[(2 k) ! / 2] \cdot Z$ for $k$ odd .

A related result concerns the stable homotopy of complex projective spaces:

THEOREM 1.2. Let $\mathrm{CP}^{k}$ denote $k$-dimensional complex projective space (2k real dimensions). Let $\mu_{k}$ be, as in [1], the generator of a $Z_{2}$ summand of $\pi_{8 k+1}^{S}\left(S^{0}\right)$ which is represented in the Adams spectral sequence by $P^{k} h_{1}$. Let $1_{2}$ denote the integral generator of $\pi_{2}^{S}\left(\mathrm{CP}^{k}\right)$. Then $\mu_{n} 1_{2}$ is nonzero in $\pi_{8 n+3}^{S}\left(\mathrm{CP}^{4 n+1}\right)$ and is zero in $\pi_{8 n+3}^{S}\left(\mathrm{CP}^{4 n+2}\right)$.

2. Some standard results. Let $y$ generate $H^{4}\left(\mathrm{QP}^{\infty} ; Z\right)$. Let $\eta$ be the standard quaternionic line bundle over $\mathrm{QP} \mathrm{P}^{\infty}, \eta-2=\mu \in K U^{0}\left(\mathrm{QP}^{\infty}\right)$. Let $h(k)$ be the positive integer such that the image of the stable Hurewicz homomorphism in $H^{4 k}\left(\mathrm{QP}^{\infty} ; Z\right)=Z$ is $h(k) \cdot Z$. Since (cf. Mosher, [2]) the image of the stable Hurewicz homomorphism in $H_{4 k}\left(\mathrm{CP}^{\infty} ; Z\right)=Z$ is $(2 k) ! \cdot Z$ it is clear that $h(k)$ divides $(2 k) !$. On the other hand using the fact that $c h \mu=\sum_{n=1}\left[2 y^{n} / n !\right]$ it is clear that $(2 k) ! / 2$ divides $h(k)$. For $k$ even this last statement can be strengthened by a factor of 2 so that $(2 k)$ ! divides $h(k)$; one exploits the fact

Received by the editors August 11, 1969.

AMS Subject Classifications. Primary 5540, 5552.

Key Words and Phrases. Complex projective space, quaternionic projective space, Hurewicz homomorphism, Adams spectral sequence.

: This research was supported in part by National Science Foundation Grant GP-9587. 
that, for $k$ even, the natural reduction map $K S p^{0}\left(S^{4 k}\right) \rightarrow K U^{0}\left(S^{4 k}\right)$ is a doubling map. To prove Theorem 1.1 it remains only to show that for $k$ odd $h(k)$ is $(2 k) ! / 2$ and not $(2 k)$ !.

3. Remarks on $\operatorname{Ext}_{A}\left(\widetilde{H}^{*}\left(\mathrm{CP}^{n} ; Z_{2}\right), Z_{2}\right)$. Let $E_{r}(X)$ denote the $r$ th term of the Adams spectral sequence for $\pi_{*}^{S}(X ; 2)$, the 2-primary component of the stable homotopy of $X . E_{r}(X)$ is an $E_{r}\left(S^{0}\right)$ left module and $E_{2}^{s, t}(X)$ is just $\operatorname{Ext}_{A}^{s, t}\left(\widetilde{H}^{*}\left(X ; Z_{2}\right), Z_{2}\right)$ where $A$ is the $\bmod$ 2 Steenrod algebra.

We filter $E_{2}\left(\mathrm{CP}^{n}\right), n \leqq \infty$, by setting $F^{2 k} E_{2}\left(\mathrm{CP}^{n}\right)$ to be the image of $E_{2}\left(\mathrm{CP}^{k}\right)$ for $k<n$ and $E_{2}\left(\mathrm{CP}^{n}\right)$ for $k \geqq n ; F^{2 k+1}=F^{2 k}$. We may then choose generators for $E_{2}\left(\mathrm{CP}^{n}\right)$ denoted by symbols of the form ${ }_{2 k} g$ so that $g$ is a generator of $E_{2}^{s, t-2 k}\left(S^{0}\right)$ and ${ }_{2 k} g$ is of exact filtration $2 k$ and comes from an element of $E_{2}^{s, t}\left(\mathrm{CP}^{k}\right)$ which under the induced map of the pinching map $\mathrm{CP}^{k} \rightarrow S^{2 k}$ goes into $g \cdot 1_{2 k}$ in $E_{2}^{s, t}\left(S^{2 k}\right)$ where $1_{2 k}$ generates $E_{2}^{0,2 k}\left(S^{2 k}\right)$.

We are interested particularly in two sorts of generators in $E_{2}\left(\mathrm{CP}^{n}\right)$ :

(i) The ' $Z$-towers'; and

(ii) Certain elements of filtration 2 and 4 on the 'top edge'.

The ' $Z$-towers': A $Z$-tower is a family of elements $h_{0}^{n} \cdot b$ which are nonzero for all $n \geqq 0$ and such that $b \neq h_{0} \cdot b^{\prime}$. There is just one $Z$ tower in the $2 k$-stem of $E_{2}\left(\mathrm{CP}^{\infty}\right)$; it consists precisely of the elements of exact filtration $2 k$. All of the tower, save for a possible finite segment at the bottom, persists to $E_{\infty}\left(\mathrm{CP}^{\infty}\right)$ and represents a subgroup of the integral summand of $\pi_{2 s}^{S}\left(\mathrm{CP}^{\infty} ; 2\right)$. But since multiplication by 2 (or any other element of $\pi_{*}^{S}\left(S^{0} ; 2\right)$ ) cannot increase exact filtration this subgroup of the integral summand turns out to be the entire summand. We may therefore say that the $Z$-tower in the $2 k$-stem of $E_{2}\left(\mathrm{CP}^{\infty}\right)$ is generated by a bottom element of the form ${ }_{2 k} h_{0}^{d(k)}$ where $d(k) \leqq k-\alpha(k)$ and that just those elements of the tower of the form $h_{0}^{n} \cdot{ }_{2 k} h_{0}^{d(k)}$ with $n \geqq k-\alpha(k)-d(k)$ will persist to $E_{\infty}(\mathrm{CP} \infty)$. Here $\alpha(k)$ stands for the sum of the digits of the dyadic expansion of $k$. (Conjecture: If $2^{s-1} \leqq k<2^{s}$ then $d(k)=2^{s}-k-1$.)

Since $\mathrm{CP}^{\infty}$ is an $H$-space, $E_{r}\left(\mathrm{CP}^{\infty}\right)$ and $\pi_{*}^{S}\left(\mathrm{CP}^{\infty} ; 2\right)$ have product structures; $\pi_{*}^{S}\left(\mathrm{CP}^{\infty} ; 2\right) /$ torsion is a polynomial ring over the integers (without identity) generated by $1_{2}$ (cf. [2, Theorem 2.1]). Then for $m, n$ such that $\alpha(m+n)=\alpha(m)+\alpha(n)$ we must have

$$
\begin{aligned}
\left(h_{0}^{m-\alpha(m)-d(m)} \cdot{ }_{2 m} h_{0}^{d(m)}\right) & \left(h_{0}^{n-\alpha(n)-d(n)} \cdot{ }_{2 n} h_{0}^{d(n)}\right) \\
& =h_{0}^{m+n-\alpha(m+n)-d(m+n)} \cdot 2(m+n) h_{0}^{d(m+n)} ;
\end{aligned}
$$

in words, the $2 m$-stem $Z$-tower times the $2 n$-stem $Z$-tower gives the 
$2(m+n) Z$-tower when there is no dyadic carryover in adding $m$ to $n$. The proof has been in $E_{\infty}\left(\mathrm{CP}^{\infty}\right)$ but the same is true in $E_{r}\left(\mathrm{CP}^{\infty}\right)$, $r<\infty$.

The 'top edge': The maps $S^{2} \stackrel{i}{\rightarrow} \mathrm{CP}^{2} \stackrel{p}{\rightarrow} S^{4}$ induce a short exact sequence in cohomology and so a long exact sequence

$$
\rightarrow E_{2}^{s, t}\left(\mathrm{CP}^{2}\right) \stackrel{p_{*}}{\rightarrow} E_{2}^{s, t}\left(S^{4}\right) \stackrel{\partial}{\rightarrow} E_{2}^{s+1, t}\left(S^{2}\right) \stackrel{i_{*}}{\rightarrow} E_{2}^{s+1, t}\left(\mathrm{CP}^{2}\right) \rightarrow
$$

where $\partial$ is given by $\partial\left(g \cdot 1_{4}\right)=h_{1} g \cdot 1_{2}$.

We have well-defined generators

$$
{ }_{2} P^{p} h_{1} \in E_{2}^{4 p+1,12 p+4}\left(\mathrm{CP}^{2}\right), \quad{ }_{4} P^{p-1} h_{0}^{3} h_{3} \in E_{2}^{4 p, 12 p+3}\left(\mathrm{CP}^{2}\right)
$$

for $p \geqq 1$; they are the two 'highest' elements (in value of $s$ ) in the $8 p+3$-stem of $E_{2}\left(\mathrm{CP}^{2}\right)$ and their images in $E_{2}\left(\mathrm{CP}^{k}\right), k \leqq 4 p+1$, are the two highest elements in the $8 p+3$ stem there.

Lemma. In $E_{2}\left(\mathrm{CP}^{k}\right), h_{0} \cdot{ }_{4} P^{p-1} h_{0}^{3} h_{3}={ }_{2} P^{p} h_{1}, k \leqq 4 p+1$.

Proof. Write down actual resolutions for $k=2$.

\section{The main theorems.}

Theorem 4.1. If $2 n=4(\bmod 8), \alpha(n)=r, n \neq 2$, then in $E_{r}\left(\mathrm{CP}^{\infty}\right)$, $d_{r}\left({ }_{2 n} h_{0}^{r-1}\right)={ }_{2} P^{(n-2) / 4} h_{1}$.

Proof. By induction on $r$. Let $r=2$, (the minimum value) $2 n=2^{k}+4, k \geqq 3$, and let us examine $E_{*}\left(\mathrm{CP}^{n}\right)$. We have a long exact sequence

$$
\cdots \rightarrow E_{2}^{s, t}\left(\mathrm{CP}^{n}\right) \rightarrow E_{2}^{s, t}\left(S^{2 n}\right) \stackrel{\delta}{\rightarrow} E_{2}^{s+1, t}\left(\mathrm{CP}^{n-1}\right) \rightarrow E_{2}^{s+1, t}\left(\mathrm{CP}^{n}\right) \rightarrow \cdots
$$

Suppose there is no nonzero ${ }_{2 n} h_{0}^{n-3} \in E_{2}^{n-3.3 n-3}\left(\mathrm{CP}^{n}\right)$. This would imply $\delta\left(h_{0}^{n-3} \cdot 1_{2 n}\right) \neq 0$, and then

$$
\delta\left(h_{0}^{n-3} \cdot 1_{2 n}\right)={ }_{4} P^{(n-6) / 4} h_{0}^{2} h_{3}
$$

and

$$
\delta\left(h_{0}^{n-2} \cdot 1_{2 n}\right)={ }_{2} P^{(n-2) / 2} h_{1}
$$

and therefore there could be no nonzero element ${ }_{2 n} h_{0}^{n-2} \in E_{2}\left(\mathrm{CP}^{n}\right)$; this is impossible and so, in fact $0 \neq_{2 n} h_{0}^{n-3} \in E_{2}^{n-3.3^{n}-3}\left(\mathrm{CP}^{n}\right)$. But since in fact this element must be killed by the time we get to $E_{\infty}$ we must have

$$
d_{2}\left({ }_{2 n} h_{0}^{n-3}\right)={ }_{2} P^{(n-2) / 4} h_{1}
$$


Now let $n$ be given such that $2 n \equiv 4(\bmod 8), \alpha(n)=r, n=n^{\prime}+n^{\prime \prime}$ where $n^{\prime}$ is the highest power of 2 less than $n$ and, accordingly, $\alpha\left(n^{\prime \prime}\right)=r-1$. In $E_{r-1}\left(C P^{\infty}\right)$ we have that $2 n^{\prime} h_{0}^{n^{\prime}-1} \neq 0$ and, by induction, we may assume that ${ }_{2 n^{\prime \prime}} h_{0}^{n^{\prime \prime}-r} \neq 0$. Hence ${ }_{2 n} h_{0}^{n-r-1} \neq 0$. There are two possibilities for eliminating ${ }_{2 n} h_{0}^{20-r-1}$;

(i) $d_{r+1}\left(2 n h_{0}^{n-r-1}\right)={ }_{4} P^{(h-6) / 4} h_{0}^{3} h_{3}$,

(ii) $d_{r}\left(2 n h_{0}^{n-r-1}\right)={ }_{2} P^{(n-2) / 4} h_{1}$.

Possibility (i) would imply however that $d_{r-1}\left({ }_{2 n} h_{0}^{n-r}\right)={ }_{2} P^{(n-2) / 4} h_{1}$ which is impossible. We have therefore that ${ }_{2 n} h_{0}^{n-r-1}$ persists to $E_{r}$ where it is killed. This concludes the proof of Theorem 4.1. Theorem 1.2 is an immediate corollary.

Let us now consider $E_{*}\left(Q^{\infty}\right)$ using the same sort of terminology as before. We wish to show what happens to the $Z$-tower in the $2 n$ stem, $2 n \equiv 4(\bmod 8)$. Specifically we are interested in knowing whether ${ }_{2 n} h_{0}^{n-r-1}$ persists to $E_{\infty}, r=\alpha(n)$. We know that ${ }_{2 n} h_{0}^{n-r-1}$ is nonzero in $E_{r}\left(\mathrm{CP}^{\infty}\right)$ and so also in $E_{r}\left(\mathrm{QP}^{\infty}\right)$. But in $E_{r}\left(\mathrm{QP}^{\infty}\right)$ this element is trapped, there being no element ${ }_{2} P^{(n-2) / 4} h_{1}$ to send ${ }_{2 n} h_{0}^{n-r-1}$ into under $d_{r}$ and certainly nothing with higher $s$-filtration. We have proved Theorem 1.1.

\section{REFERENCES}

1. M. Mahowald, The metastable homotopy of $S^{n}$, Mem. Amer. Math. Soc., No. 72 (1967).

2. R. E. Mosher, Some stable homotopy of complex projective spaces, Topology 7 (1968), 179-193. MR 37 \#3569.

University of Illinois at Chicago Circle, Chicago, Illinois 60680 